\title{
An Electrochemical Enzyme Immunochip Based on Capacitance Measurement for the Detection of IgG
}

\author{
Seung Jae Yi, Ji Hye Choi, ${ }^{\dagger}$ Hwa-Jung Kim, ${ }^{\ddagger}$ Seung-Cheol Chang, ${ }^{\dagger, *}$ \\ Deog-Su Park, ${ }^{\dagger}$ Kyung Chun Kim, and Chulhun L. Chang ${ }^{\S, *}$ \\ Multiscale Multiphysics Measurement Laboratory, School of Mechanical Engineering, \\ Pusan National University, Busan 609-735, Korea \\ ${ }^{\dagger}$ Institute of BioPhysio Sensor Technology, Pusan National University, Busan 609-735, Korea. *E-mail: s.c.chang@pusan.ac.kr \\ Department of Microbiology and Research Institute for Medical Science, College of Medicine, \\ Chungnam National University, Daejeon 305-764, Korea \\ ${ }^{\S}$ Department of Laboratory Medicine, School of Medicine, and Medical Research Institute, Pusan National University, \\ Busan 609-735, Korea. *E-mail: cchl@medimail.co.kr \\ Received February 10, 2011, Accepted February 21, 2011
}

\begin{abstract}
This study describes the development of an electrochemical array immunochip for the detection of IgG. Interdigitated immunochip platforms were fabricated by sputtering gold on a glass wafer by using MEMS process and then were coated with Eudragit S100, an enteric polymer, forming an insulating layer over the working area of immunochips. The breakdown of the polymer layer was exemplified by the catalytic action of urease which, in the presence of urea, caused an alkaline $\mathrm{pH}$ change. This subsequently caused an increase of the double layer capacitance of the underlying electrode. Used in conjunction with a competitive immunoassay format, this allowed the ratio of initial to final electrode capacitance to be directly linked with the concentration of analyte, i.e. IgG. Responses to IgG could be detected at IgG concentration as low as $250 \mathrm{ngmL}^{-1}$ and showed good linearity up to IgG concentration as high as $20 \mu \mathrm{gmL}^{-1}$.
\end{abstract}

Key Words : Immunochip, Enzyme immunoassay, Capacitance, Impedometric, Interdigitated electrode

\section{Introduction}

Electrochemical impedance spectroscopy (EIS) is a unique technique for the study of both bulk and interfacial electrical characteristics and processes of electrode systems in an electrolyte. ${ }^{1,2}$ Originally, EIS has been used for non-biological systems such as protective coatings over metal substance, corrosion studies or fuel cell diagnostics. ${ }^{3-6}$ During the last two decades EIS is frequently applied to sensor based immunochip systems coupled with functional polymers such as $\mathrm{pH}$ sensitive polymers. Impedance based biochips are usually consist of a dielectric, chemically sensitive film coated onto a substrate electrode. The film passes a low conduction current making amperometric or conductimetric measurements less sensitive for signal transduction. The analyte is detected by measurement of changes in the films capacitive properties associated with its dielectric constant, charge uptake or formation of interface dipole layers, when an active species is introduced. The capacitance of the underlying electrode is determined by the dielectric properties and thickness of the insulating layer, therefore a change in the dielectric causes a measurable change in the capacitance. ${ }^{7}$

Enzyme immunosensors typically consist of an antibody coated electrode which is brought into contact with either a mixture of antigen and enzyme-labeled antigen for competitive binding to the antibody, or sequentially exposed to the antigen and an enzyme-labeled antibody, i.e., sandwich type assays. The degree of electrode membrane activation by enzyme can be estimated from the electrode signal in the presence of substrate. There have been, however, limited studies on this type of immunosensor coupled with capacitance or impedance measurement, ${ }^{8-10}$ which may be due to difficulties in linking an enzyme reaction to a method for changing the impedance or capacitance of an electrode. McNeil et al. introduced ability of sensor based on the measurement of impedance change by using polymer coatings on electrodes and analysis the antigen-antibody reaction qualitatively by immunostrips and quantitatively by EIS. ${ }^{11-15}$

The work presented in this study aims to utilize the measurement of the double layer capacitance of coated electrodes in conjunction with competitive immunoassay techniques with a view to the development of immunosensors for the measurement of clinically relevant analytes. This will be achieved by introducing a new immunochip system based on the measurement of capacitance changes of a $\mathrm{pH}$-sensitive polymer, Eudragit $\mathrm{S} 100$, which is widely used in clinical and pharmaceutical studies. ${ }^{16-18}$ The $\mathrm{pH}-$ sensitive polymer was layered and then formed an insulating layer by dip-coating procedure on the surface of the interdigitated electrodes. The breakdown of the polymer layer was initiated by the reaction of urea with urease at the electrode surface and subsequently caused an increase of the double layer capacitance of the underlying electrode. This capacitance change was directly used for the selective and sensitive detection of IgG. The Interdigitated chips were fabricated by MEMS process and results much smaller than 
other conventional screen printing interdigitated chips. Investigations into optimization of electrochemical immunochip configurations were performed to develop a rapid, sensitive and specific technique for the measurement of IgG.

\section{Experimental}

Reagents and Materials. ProLinker, one of the calixarene derivatives with crown-ether moiety, was purchased from Proteogen (Korea) for use as a linker system for immobilization of antibody. A pH sensitive polymer hydrogel, Eudragit S100 (methacrylic acid copolymer type B) was obtained from Dumas Limited (UK). Urease from Canavalia ensiformis (Jack bean), human anti-rabbit immunoglobulin G (anti$\mathrm{IgG}$ ), immunoglobulin $\mathrm{G}$ ( $\mathrm{IgG})$, bovine serum albumin (BSA), disodium hydrogen phosphate, sodium dihydrogen phosphate, sodium chloride, ethylenediaminetetra-acetic acid dipotassium salt (EDTA), ethanol and urea were obtained from Sigma Aldrich (USA). A phosphate buffer saline solution (PBS) was prepared by modifying $0.1 \mathrm{M}$ of disodium hydrogen phosphate with the mixture of $0.1 \mathrm{M}$ of sodium dihydrogen phosphate with $0.1 \%$ sodium chloride. All other chemicals were of extra pure analytical grade and used without further purification. All aqueous solutions were prepared in doubly distilled water, which was obtained from a Milli-Q water purifying system (USA).

Preparation of IgG-urease Conjugate. To perform a competitive immune-binding between IgG and IgG-urease conjugate, IgG-urease conjugate has been prepared; $5 \mathrm{mg}$ of urease was dissolved in $1 \mathrm{~mL}$ of $100 \mathrm{mM}$ PBS (pH 7.4). A $0.2 \mathrm{~mL}$ aliquot of $25 \%$ glutaraldehyde was mixed with the urease solution and the mixture was incubated at $25{ }^{\circ} \mathrm{C}$ for 18 hours. Excess glutaraldehyde was then removed by passing the mixture through a Sephadex G-25 column equilibrated with $0.9 \% \mathrm{NaCl}$. The mixture was then added to $1 \mathrm{mgmL}^{-1} \mathrm{IgG}$ and left to incubate for 24 hours at $4{ }^{\circ} \mathrm{C}$, following which, blocking of $\operatorname{IgG}$ was done with $1 \%$ BSA solution for 2 hours followed by overnight dialysis against PBS (pH 7.4). The conjugate solution was filtered through a sterile Millipore membrane (pore size $0.2 \mu \mathrm{m}$ ) and the filtrate was stored at $-20{ }^{\circ} \mathrm{C}$ until use. A standard urease activity assay was performed and the concentration of the urease conjugate was estimated using the method reported previously. ${ }^{20,21}$

Interdigitated Array Chip Preparation. Comb type interdigitated gold array chips were designed and manufactured by using a standard MEMS process at Pusan National University's MEMS facility. As can be seen in Figure 1, each chip has $10 \mathrm{~mm} \times 10 \mathrm{~mm}$ comb type electrode area and the width of electrode and the gap between electrodes are $100 \mu \mathrm{m}$.

Preparation of Immunosensor. To immobilize antibody onto the array chip ProLinker-SAM has been carried out. A bare gold chip fabricated was cleaned with a Piranha solution. ${ }^{19}$ An aliquot of $0.2 \mathrm{mM}$ ProLinker solution was prepared in formaldehyde and the SAM was formed by immersion of the gold chip into $0.2 \mathrm{mM}$ ProLinker solution

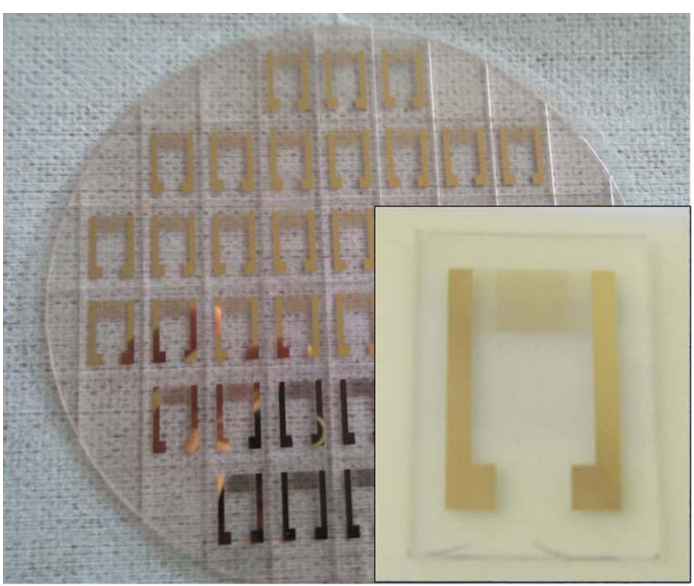

Figure 1. Interdigitated immunochips, the fabrication and the dimensional details of which are given in the Experimental Section.

and the chip was rinsed with chloroform-methanol mixture solution $(1: 100)$ and then with methanol for 10 minutes. The chip then immersed into $250 \mu \mathrm{gmL}^{-1}$ anti-IgG solution to allow antibody binding onto the ProLinker-SAM layer for 8 $\mathrm{h}$ at $4{ }^{\circ} \mathrm{C}$. After incubation, the chip was removed and was gently rinsed using PBS. The chip surface was then blocked with $0.1 \%$ BSA solution by immersing the chip for 15 minutes and was then rinsed thoroughly by PBS. The antibody modified chip was incubated in the mixture of IgG and IgG-urease conjugate solution at $25^{\circ} \mathrm{C}$ for $10 \mathrm{~min}$ and then rinsed in the washing buffer (50 mM PBS, $\mathrm{pH}$ 7.0). The chips then gently dried by medical tissue and softly dried in a nitrogen stream for 15 minutes at $25^{\circ} \mathrm{C}$. Subsequently, the chips were simply dipped in the $10 \%(\mathrm{w} / \mathrm{v}) \mathrm{S} 100$ polymer solution prepared in methoxy propoxy propanol. After the dip-coating, chips were laid flat for 10 minutes to allow settling of the polymer layer before drying for 1 hour at 25 ${ }^{\circ} \mathrm{C}$.

Electrochemical Measurement Procedure. In order to study the $\mathrm{pH}$ profile of the polymer coated chips, impedance measurements with a full frequency sweep were performed at zero DC potential with respect to the counter electrode, with an AC peak to peak amplitude of $30 \mathrm{mV}$ using a 5 seconds integration time and a comparative resistor of 10 $\mathrm{k} \Omega$ Impedance spectra were recorded over the frequency range $20 \mathrm{kHz}$ to $150 \mathrm{KHz}$ by using an electrochemical workstation, VersaStat3 (AmeTek, USA). $10 \mathrm{~mL}$ solutions of $100 \mathrm{mM} \mathrm{NaCl}$ containing $0.2 \mathrm{mM}$ EDTA were adjusted to $\mathrm{pH}$ ranged from 5.0 to 9.0 using $0.1 \mathrm{M} \mathrm{HCl}$ and $0.1 \mathrm{M}$ $\mathrm{NaOH}$ and were used as operating electrolytes. Measurements were performed in two electrode mode using a glassy carbon electrode as a counter electrode. This was placed directly opposite and parallel to the working electrode with a separation of approximately $3 \mathrm{~mm}$ and ensuring that the interdigitated electrode stems made no contact with electrolyte. For immunosensing of IgG, the electrochemical cell setup was the same as that used for the full frequency sweep but in $5.0 \mathrm{~mL}$ of $50 \mathrm{mM}$ PBS (pH 6.5) containing target analyte. A $500 \mu \mathrm{L}$ aliquot of urea standards prepared in 280 $\mathrm{mM} \mathrm{NaCl}$ containing $2 \mathrm{mM}$ EDTA (pH 5.5) was added to 
initiate the polymer breakdown and the impedance measurement was performed at $20 \mathrm{kHz}$.

\section{Results and Discussion}

Sensing Chemistry. A schematic diagram of the immunosensing concept is illustrated in Figure 2. As can be seen, the anti-IgG modified on the surface of the gold layer binds with target antigen, IgG. Unknown amount of sample IgG, however, can be quantified after competition with the IgG-ureaseconjugate. Urease modified on the conjugate then reacts with urea and produce $\mathrm{NH}_{3}$. The enzymatic production of $\mathrm{NH}_{3}$ increases local $\mathrm{pH}$ above the surface of the polymer, Eudragit S100 layer and initiates the polymer breakdown. The polymer layer passes a low conduction current making conductimetric measurements less sensitive for signal transduction. The analyte, IgG, then can be detected by measurement of changes in the polymer layer capacitive properties associated with its dielectric constant, charge uptake or formation of interface dipole layers, when an active species is introduced. The capacitance of the underlying electrode is determined by the dielectric, therefore a change in the dielectric causes a measurable change in the capacitance. ${ }^{7}$

pH Profile Using Interdigitated Electrodes. Figure 3

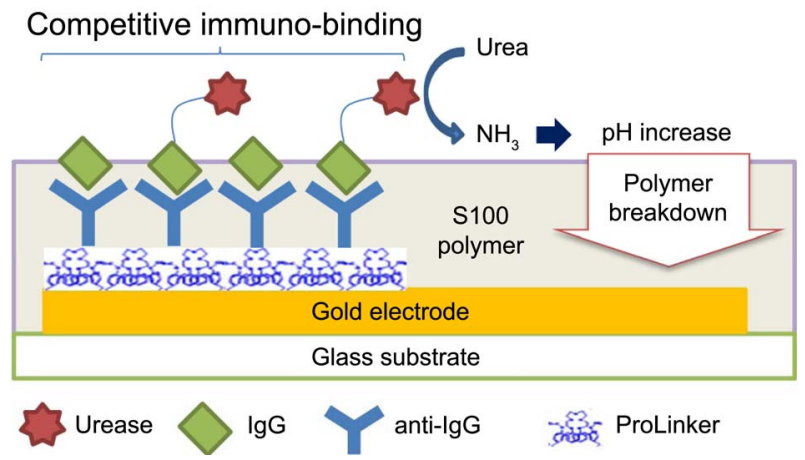

Figure 2. Schematic representation of competitive immunosensing of IgG by using an interdigitated immunochip.

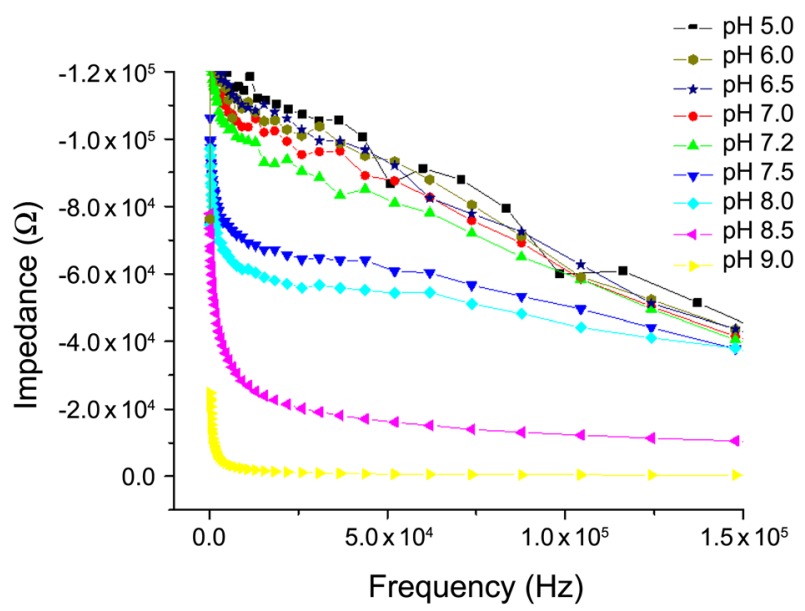

Figure 3. Polymer degradation evaluation with $\mathrm{pH}$ through impedance changes. Impedance spectra were recorded over the frequency range $20 \mathrm{kHz}$ to $150 \mathrm{KHz}$ in $100 \mathrm{mM} \mathrm{NaCl}$ containing $0.2 \mathrm{mM}$ EDTA and adjusted to $\mathrm{pH}$ ranged from 5.0 to 9.0 . shows $\mathrm{pH}$ effect on the polymer coated chips using the method described in the Experimental Section. Upon increase in $\mathrm{pH}$ of the applied solution, an increase in overall impedance change of the electrode was apparent, which confirmed expectations and proved that the polymer coating was sensitive to $\mathrm{pH}$. Very little breakdown of the polymer was seen for solutions of $\mathrm{pH}$ below 7, which again was expected due to the $\mathrm{pH}$ sensitive polymer being known to be dissolve at any $\mathrm{pH}$ higher than $7 .{ }^{16}$ These results therefore allowed further investigations into polymer breakdown to be initiated using the reaction between urea and urease to cause the increase in $\mathrm{pH}$ which would cause the polymer to dissolve.

Capacitance Calculation in the Present System. According to interpreting AC impedance, it is possible to substitute an electric circuit for phenomenon at the interface between metal and solution. This electric circuit is called equivalent circuit and electrochemical characteristics about the experimental system can be interpreted by proper equivalent circuit. $^{22}$ In Figure 4(a) shows equivalent circuit between bare electrode surface and the solution. It is basic model about the interface between metal and solution. It is composed with three electrical parameters; electrolyte resistance $\left(R_{e}\right)$, the charge-transfer resistance $\left(R_{c t}\right)$ and the double-layer capacitance $\left(\mathrm{C}_{\mathrm{dl}}\right)$. In this case, the impedance value of circuit is expressed by below equation (1). ${ }^{22,23}$

$$
Z(\omega)=R_{e}+\frac{R_{c t}}{1+\omega^{2} R_{c t}^{2} C_{d l}^{2}}-\frac{j \omega R_{c t}^{2} C_{d l}}{1+\omega^{2} R_{c t}^{2} C_{d l}^{2}}
$$

In this study, the equivalent circuit should be modified because the electrode is coated by the $\mathrm{S} 100$ polymer. Figure 4(b) represents a modified equivalent circuit to describe the effect of coated polymer on the electrode. Compared with the basic model, some electrical parameters are added to describe the polymer effect; the geometric capacitance of the polymer $\left(\mathrm{C}_{\mathrm{g}}\right)$ and the bulk resistance $\left(\mathrm{R}_{\mathrm{b}}\right)$. The impedance value of modified equivalent circuit is expressed by equation (2);

$$
Z(\omega)=R_{e}+\frac{C D-B E}{D^{2}+E^{2}}-j\left(\frac{B D+C E}{D^{2}+E^{2}}\right)
$$

when $\mathrm{E}=\omega \mathrm{C}_{\mathrm{g}} \mathrm{C}, \mathrm{D}=1+\omega \mathrm{C}_{\mathrm{g}} \mathrm{B}, \mathrm{C}=\mathrm{R}_{\mathrm{b}}+\mathrm{A}, \mathrm{B}=\omega \mathrm{C}_{\mathrm{dl}} \mathrm{R}_{\mathrm{ct}}{ }^{2} /$ $\left(1+\omega^{2} \mathrm{C}_{\mathrm{dl}}{ }^{2} \mathrm{R}_{\mathrm{ct}}{ }^{2}\right)$ and $\mathrm{A}=\mathrm{R}_{\mathrm{ct}} /\left(1+\omega^{2} \mathrm{C}_{\mathrm{dl}}{ }^{2} \mathrm{R}_{\mathrm{ct}}{ }^{2}\right)$.

There are many electrical parameters, but the geometric capacitance of the polymer $\left(\mathrm{C}_{\mathrm{g}}\right)$ is the most sensitive and stable value to describe the characteristic change of polymer. ${ }^{22}$ If the geometrical characteristic of polymer were changed by the external reaction, the $C_{g}$ value is changed and there are three orders of magnitude difference between $\mathrm{C}_{\mathrm{g}}$ and $\mathrm{C}_{\mathrm{dl}}$.

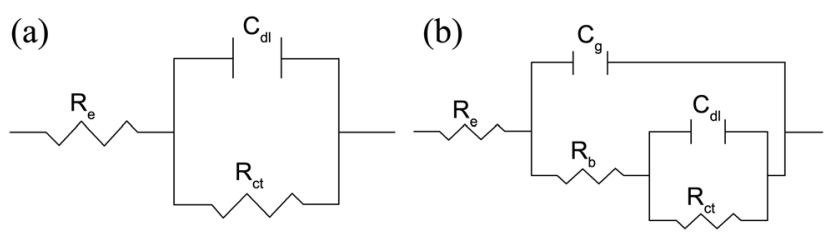

Figure 4. Equivalent circuits for the impedance behavior of an electrode: (a) bare electrode and (b) polymer coated electrode. 
The geometric capacitance of the polymer $\left(\mathrm{C}_{\mathrm{g}}\right)$ can be calculated from Bode plot $(\log |Z|-\log (\omega))$. From Bode plot of $\log |Z|-\log (\omega), \log \left(R_{e}+R_{b}\right)$ value could be calculated at the low-frequency and $\log \left(\mathrm{R}_{\mathrm{e}}\right)$ value can be described at the high-frequency. The equation (3) could be earned at the between of low and high frequency through some simplification and equation (4) shows the log scale on both side of equation (3).

$$
\begin{gathered}
Z(\omega) \cong \frac{1}{\omega C_{g}} \\
\log (Z(\omega))=-\log (\omega)-\log \left(C_{g}\right)
\end{gathered}
$$

The $y$-intercept of equation (4) represents the $\mathrm{C}_{\mathrm{g}}$ parameter, so we can calculate the capacitance $\left(\mathrm{C}_{\mathrm{g}}\right)$ value from Bode plot and equation (4).

Breakdown of Polymer Coating by Enzymatic Reaction. Each experiment was set up in $5.0 \mathrm{~mL}$ of $50 \mathrm{mM}$ PBS $(\mathrm{pH}$ 6.5 ). The polymer breakdown was initiated by addition of $10 \mathrm{mM}$ urea standard prepared as described in the Experimental Section. The capacitance changes obtained from interdigitated chips with polymer breakdown as a function of urea concentration were apparently observed. The capacitance responses increased as urea concentration increased from 0 to $10 \mathrm{mM}$ and then reached a plateau above $10 \mathrm{mM}$. The capacitance responses did not change significantly between 10 and $30 \mathrm{mM}$. Therefore, the urea concentration was fixed at $10 \mathrm{mM}$ for subsequent experiments. Figure 5 shows typical assay results for the chips with IgG-ureaseconjugate standard without target IgG. The plot illustrates that the constructs were enabling distinction between the three conjugate solutions within 20 minutes of total assay time. It was indicated that there was no detectable capacitance change measured for the blank and the breakdown of the polymer layer was not initiated without the IgG-urease conjugate. Additionally, the result also confirmed that no detectable non-specific binding was occurred in the present system. As reported previously, the detection principle was realized according to the competitive properties of urease as

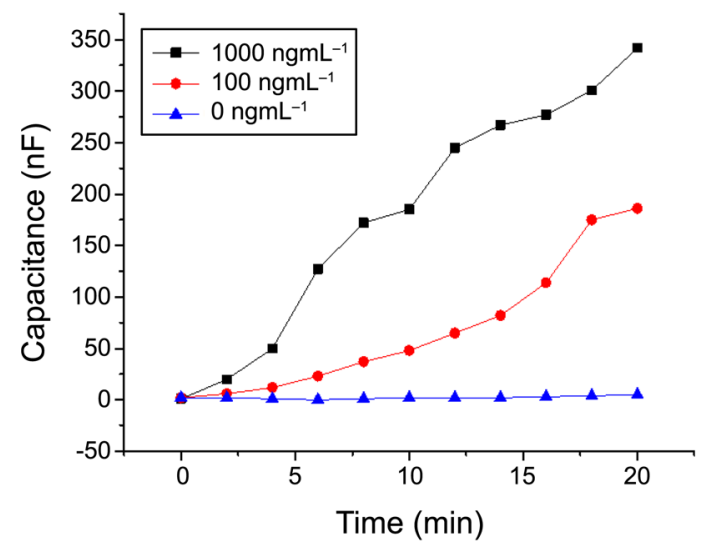

Figure 5. Capacitance changes calculated from electrochemical measurements using interdigitated immunochips bound to 0,100 and $1000 \mathrm{ngmL}^{-1}$ IgG-urease-conjugate standards. Each measurement was carried out at $20 \mathrm{kHz}$ in $50 \mathrm{mM}$ PBS (pH 6.5) containing $10 \mathrm{mM}$ urea standard. a pH-shifting marker enzyme. ${ }^{24}$ It is also note that the urease reaction is rapid and yields sharper endpoints, when compared with other enzymes such as peroxidase, since there is virtually no product inhibition associated with urea conversion to ammonia. ${ }^{24-26}$ Further experiments have been performed with higher concentrations of the conjugate to determine the optimum condition for the unconjugated $\mathrm{IgG}$ quantification. The optimum concentration of the conjugate was found to be $1.0 \mu \mathrm{gmL}^{-1}$ since there were no significant increases with the conjugate concentrations higher than 1.0 $\mu \mathrm{gmL}^{-1}$. All experiments for the construction of $\operatorname{IgG}$ calibration curves were carried out at this condition.

Impedometric Response of Array Chip. In order to achieve optimum array chip performance conditions, the effect of $\mathrm{pH}$ and buffer nature were investigated. The optimum $\mathrm{pH}$ and buffer composition were assessed using the conjugate concentration of $1.0 \mu \mathrm{gmL}^{-1}$ in four different buffer solutions; $50 \mathrm{mM}$ and $100 \mathrm{mM}$ PBS (pH 4.0-7.0), 50 $\mathrm{mM}$ glycine buffer ( $\mathrm{pH} 4.0-7.0)$ and $50 \mathrm{mM}$ Tris/HCl buffer (pH 4.0-7.0). The maximum response was obtained and found to be in 50 and $100 \mathrm{mM}$ PBS at $\mathrm{pH}$ 5.5-6.5. $50 \mathrm{mM}$ Phosphate buffer ( $\mathrm{pH}$ 6.5) was chosen as the operating buffer after consideration of the optimum for enzyme activity in solution. In order to obtain a stable and highly reproducible impedometric response over a wide range of IgG concentrations, it is realized that the preconditioning of the chips is required by immersing the electrode in the operating buffer for a reasonable time before measurements. This is because the $\mathrm{S} 100$ polymer modified chips might require complete hydration before produce reproducible responses. Without hydration, inconstant initial decreases in capacitance were observed when the measurement carried out with $1.0 \mu \mathrm{gmL}^{-1}$ urease-IgG conjugate. After repeatable experiments, it was decided that a 10 minutes hydration in the operating buffer was used before initiating the enzymatic reaction by the addition of urea.

Array Chip Calibration. Capacitance responses to the array chip for IgG standard prepared in $50 \mathrm{mM}$ PBS (pH 6.5 ), were monitored. Capacitance changes obtained from

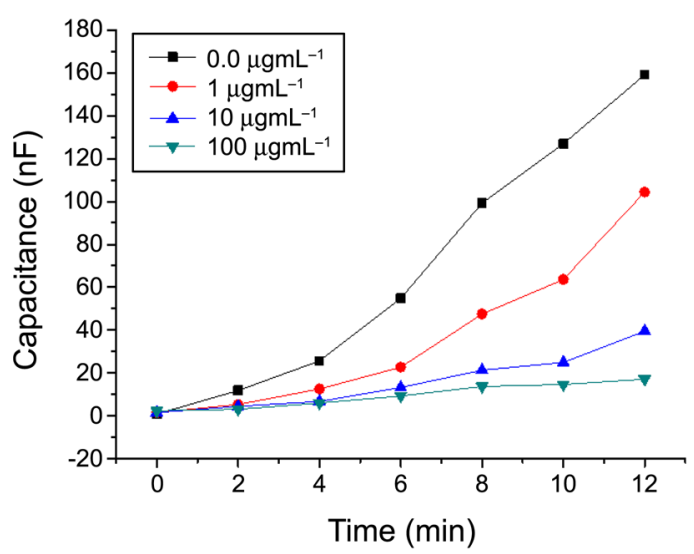

Figure 6. Capacitance responses to the immunochip for $\mathrm{IgG}$ concentrations in the range of $0.0,1.0,10.0$ and $100.0 \mu \mathrm{gmL}^{-1}$. Each measurement was carried out at $20 \mathrm{kHz}$ in $50 \mathrm{mM}$ PBS (pH 6.5) containing $10 \mathrm{mM}$ urea standard. 


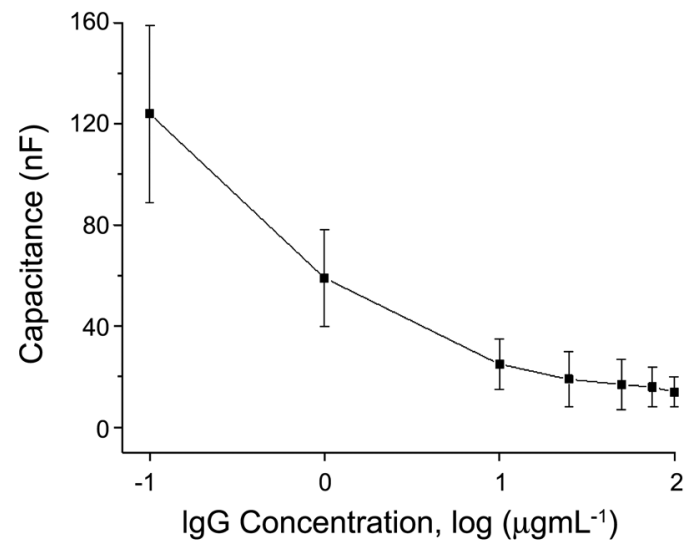

Figure 7. Calibration curve for electrochemical $\operatorname{IgG}$ detection using the immunochip. Each point represents the mean value of 5 measurements and the error bars represent one standard deviation.

IgG standard concentrations over the range of $0-100 \mu \mathrm{gmL}^{-1}$ were shown in Figure 6. Under optimized conditions, the capacitance responses obtained were on the increase shortly after the addition of urea solution and continued to increase for 20-40 minutes and reached a slight plateau. In order to construct calibration curves, the measurement procedure were modified as follows: each measurement were terminated after 10 minutes recording and the capacitance were calculated using the final highest values obtained. A calibration curve for IgG were constructed and shown in Figure 7. Each point represents the mean value of five measurements and error bars represent one standard deviation. Responses to $\mathrm{IgG}$ could be detected at $\mathrm{IgG}$ concentration as low as 0.25 $\mu \mathrm{gmL}^{-1}$ based on five-times measurement for the standard deviation of the blank noise (95\% confidence level, $k=3, n$ $=5$ ), and showed good linearity up to $\operatorname{IgG}$ concentration as high as $20 \mu \mathrm{gmL}^{-1}$. The reproducibility of the chips was investigated using IgG concentrations between $1.0 \mu \mathrm{gmL}^{-1}$ and $10 \mu \mathrm{gmL}^{-1}$. The immunochip performance demonstrated that the values of inter-chip CV (coefficient variation) were calculated to be between $13.4 \%$ and $36.7 \%$ over the whole concentrations range. It is assumed that the higher CV values could be caused by the irregularity of the polymer which was layered by the dip-coating. It is, apparently, suggested that an advanced polymer layering process such as automated ink-jet printings could improve the reproducibility of chips. These values, however, are in the range of generally accepted values reported for the IgG quantification by using electrochemical sensor systems ${ }^{27}$ and ELISA. ${ }^{28}$

\section{Conclusion}

This study has demonstrated that a separation-free impedometric immunosensing of IgG by using miniaturized interdigitated array chips constructed by MEMS procedure. The simple and highly reproducible MEMS technique and the effective protein immobilization procedure using ProLinker on the surface of gold electrodes allows inexpensive disposable immunochip manufacture which could be applied for mass-production and automated system. The interdigitated array chip coated with the $\mathrm{S} 100$ polymer reacted to different $\mathrm{pH}$ solutions as was expected, with solutions under $\mathrm{pH} 7$ having no effect on the film and those of $\mathrm{pH} 7$ and above initiating a $\mathrm{pH}$-dependent rise in the capacitance of the electrode at $20 \mathrm{kHz}$. This proved that the polymer was reacting in the expected manner when coated over the antibody/antigen modified surface and the use of urea/urease reaction to initiate polymer breakdown was of significant importance, as this would be the fundamental sensing concept in sandwich or competitive immunoassay formats widely used in clinical or pharmaceutical analysis.

Acknowledgments. This study was supported by a grant of Korea Healthcare Technology R\&D Project, Ministry for Health, Welfare \& Family Affairs, Republic of Korea (A080854).

\section{References}

1. Cortina, M.; Esplandiu, M. J.; Alegret, S.; del Valle, M.; Group, S. B. Sensor. Actuat. B-Chem. 2006, 118, 84 .

2. Tamilselvi, S.; Murugaraj, R.; Rajendran, N. Mater. Corros. 2007, $58,113$.

3. Mansfeld, F.; Liu, G.; Xiao, H.; Tsai, C. H.; Little, B. J. Corros. Sci. 1994, 36, 2063.

4. Xiao, H.; Mansfeld, F. J. Electrochem. Soc. 1994, 141, 2332.

5. Tamilselvi, S.; Murugaraj, R.; Rajendran, N. Mater. Corros. 2007, $58,113$.

6. Macdonald, D.; Sun, A. Electrochim. Acta 2006, 51, 1767.

7. Berggren, C.; Johansson, G. Anal. Chem. 1997, 69, 3651.

8. Laczka, O.; Baldrich, E.; Xavier, F.; Muñoz, F.; del Campo, F. Anal. Chem. 2008, 80, 7239.

9. de Vasconcelos, E.; Peres, N.; Pereira, C. O.; da Silva, V. Biosens. Bioelectro. 2009, 25, 870.

10. Chen, C.; Chang, K.; Chen, Y.; Lee, C.; Lee, B.; Lee, A. Biosens. Bioelectro. 2011, 26, 3072.

11. Ho, W. O.; Krause, S.; McNeil, C. J.; Pritchard, J. A.; Armstrong, R. D.; Athey, D.; Rawson, K. Anal. Chem. 1999, 71, 1940.

12. Mcneil, C. J.; Athey, D.; Ball, M.; Ho, W. O.; Krause, S.; Armstrong, R. D.; Wright, J. D.; Rawson, K. Anal. Chem. 1995, 67, 3928.

13. Fernandez, C.; McNeil, C. J.; Rawson, K. Trac.-Trend. Anal. Chem. $\mathbf{2 0 0 5}, 24,37$.

14. Fernandez, C.; McNeil, C. J.; Rawson, K.; Nilsson, O. Anal. Chem. 2004, 76, 5649 .

15. Fernandez, C.; McNeil, C. J.; Rawson, K.; Nilsson, O.; Leung, H. Y.; Gnanapragasam, V. J. Immunol. Methods 2005, 307, 1.

16. Siepmanna, F.; Siepmanna, J.; Waltherb, M.; MacRaeb, R.; Bodmeier, R. J. Controlled Release 2008, 125, 1.

17. El-Kamel, A. H.; Sokar, M. S.; Al Gamal, S. S.; Naggar, V. F. Int. J. Pharm. 2001, 220, 13.

18. Nguyen, D. A.; Fogler, H. S. Aiche. J. 2005, 51, 415.

19. Chen, H.; Lee, J.; Jo, W.-S.; Jeong, M.-H.; Koh, K. Microchim. Acta 2011, 172, 171.

20. Kaltwasser, H.; Schlegel, H. G. Anal. Biochem. 1966, 16, 132

21. Meyerhoff, M. E.; Rechnitz, G. A. Anal. Biochem. 1979, 95, 483,

22. Macdonald, J. R. Annals of Biomedical Engineer. 1992, 20, 289.

23. Chang, B. Y.; Park, S. M. Annu. Rev. Anal. Chem. 2010, 3, 207.

24. Koh, W.; Choe, E.; Lee, D.; Chang, S.; Shim, Y. Biosens. Bioelectro. 2009, 25, 211.

25. Koncki, R.; Kopczewska, E.; Glab, S. Anal. Lett. 1994, 27, 475.

26. Walcerz, I.; Koncki, R.; Leszczynska, E.; Salamonowicz, B.; Glab, S. Anal. Lett. 1996, 29, 1939.

27. Kwon, S. J.; Seo, M.; Yang, H.; Kim, S. Y.; Kwak, J. B. Korean Chem. Soc. 2010, 31, 3103.

28. Hansen, R. J.; Balthasar, J. P. J. Pharmaceut. Biomed. 1999, 21, 1011. 